\title{
Psychobiological Personality Traits Related to Sleep Disorders and Sexual Dysfunction: A Systematic Review and Meta-analysis
}

\section{Uyku Bozuklukları ve Cinsel İșlev Bozuklukları ile Illgili Psikobiyolojik Kișilik Özellikleri: Sistematik Bir Derleme ve Meta-analiz}

\author{
(D) Farzin Rezaei, (1) Azad Hemmati*, (1) Khaled Rahmani** , (D) Saeid Komasi*** \\ Neurosciences Research Center, Research Institute for Health Development, Kurdistan University of Medical Sciences, Sanandaj, Iran \\ * Faculty of Human and Social Sciences, Department of Psychology, University of Kurdistan, Sanandaj, Iran \\ * Department of Epidemiology, Liver and Digestive Research Center, Research Institute for Health Development, Kurdistan University of Medical \\ Sciences, Sanandaj, Iran \\ ** Student Research Committee, Kurdistan University of Medical Sciences, Sanandaj, Iran
}

\begin{abstract}
Sleep and its disorders are still the least understood phenomena in biology. This systematic review aims to identify four personality psychobiological models related to sleep disorders and sexual dysfunction. The literature search for English-language records was conducted on the Web of Science, Scopus, PubMed, ProQuest and Cochrane databases from January 1990 to April 2019. According to the number of studies available related to research variables and heterogeneous designs, the pooled effect size was calculated separately for both case-control and correlation studies using the random-effects method because of the heterogeneity of the studies and use of the $1^{2}$ measure. The numbers of studies on parasomnias, Restless Legs syndrome, sleep breathing disorders, daytime sleepiness and sexual dysfunction were minimal. However, the present meta-analysis on four studies on insomnia showed that the cases and controls in harm avoidance ( $\mathrm{HA})$ are significantly different $(\mathrm{p}=0.001)$. Another meta-analysis on 11 studies showed that a significant correlation exists between poor sleep quality assessed using the Pittsburgh Sleep Quality index with negative affect (NA) ( $z=10.437$, $p<0.001)$ and positive affect $(P A)(z=-6.826, p<0.001)$. In addition to the few studies on sleep and sexual disorders, the greater challenge is the wide range of these disorders, making it difficult to perform a comprehensive meta-analysis and draw a conclusion. Generally, NA/PA and HA may play critical roles in sleep quality and insomnia, respectively. These findings and limitations are debatable.
\end{abstract}

Keywords: Character, meta-analysis, personality, sexual dysfunction, sleep disorders, temperament
Öz

Uyku ve bozuklukları halen biyolojide en az anlaşılan fenomenlerden biridir. Bu sistematik derleme, uyku bozuklukları ve cinsel işlev bozukluğu ile ilgili dört kişilik psikobiyolojik modelini tanımlamayı amaçlamaktadır. Ocak 1990'dan Nisan 2019'a kadar olan Ingilizce dilindeki kayıtlar için literatür taraması Web of Science, Scopus, PubMed, ProQuest ve Cochrane veri tabanlarında gerçekleştirilmiştir. Araştırma değişkenleri ve heterojen tasarımlarla ilgili mevcut çalışma sayısına göre havuzlanmış etki büyüklüğü, heterojenlik çalışmaları, $I^{2}$ ölçüsü nedeniyle rastgele etkiler yöntemi kullanılarak hem olgu-kontrol hem de korelasyon çalışmaları için ayrı ayrı hesaplanmıştır. Parasomniler, Huzursuz Bacak sendromu, uykuda solunum bozuklukları, gündüz uykululuk ve cinsel işlev bozukluğu ile ilgili çalışma sayısı oldukça sınırlıydı. Bununla birlikte, uykusuzluk üzerine dört çalışma üzerine yapılan mevcut meta-analiz, zarardan kaçınma (harm avoidance: $\mathrm{HA}$ ) olguları ve kontrollerinin önemli ölçüde farklı olduğunu göstermiştir $(p=0,001)$. On bir çalışmanın başka bir meta-analizi, Pittsburgh Uyku Kalitesi indeksi kullanılarak değerlendirilen kötü uyku kalitesi ile negatif duygulanım (negative affect: NA) $(z=10,437, p<0,001)$ ile pozitif duygulanım (positive affect: $P A)$ arasında anlamlı bir korelasyon olduğunu göstermiştir. ( $z=-$ $6,826, p<0,001)$. Uyku ve cinsel bozukluklarla ilgili birkaç çalışmaya ek olarak, daha büyük zorluk, kapsamlı bir meta-analiz ve sonuç çıkarmayı zorlaştıran bu bozuklukların geniş yelpazesidir. Genel olarak, NA/PA ve HA sırasıyla uyku kalitesinde ve uykusuzlukta anahtar rol oynayabilir. Bulgular ve kısıtıııılar tartışmalıdır.

Anahtar Kelimeler: Karakter, meta-analiz, kişilik, cinsel işlev bozukluğu, uyku bozuklukları, mizaç

Address for Correspondence/Yazışma Adresi: Saeid Komasi, Neurosciences Research Center, Research Institute for Health Development, Kurdistan University of Medical Sciences, Sanandaj, Iran

Phone: +988334276299 E-mail: S_komasi63@yahoo.com ORCID-ID: orcid.org/0000-0002-0198-3710

Received/Geliş Tarihi: 25.01.2021 Accepted/Kabul Tarihi: 27.02.2021

${ }^{\circ}$ Copyright 2021 by Turkish Sleep Medicine Society / Journal of Turkish Sleep Medicine published by Galenos Publishing House. 


\section{Introduction}

Sleep disorders and sexual dysfunction are among the most common psychiatric morbidities in various populations around the world (1). Sleep disorders that are common in 0.4-48 percent of the United States population included insomnia, sleep breathing disorders (Central Sleep Apnea syndrome, obstructive sleep apnea, and snoring), central hypersomnolence disorders (hypersomnia and narcolepsy), parasomnias, sleeprelated movement disorders, and circadian rhythm sleep-wake disorders (2). Meanwhile, sexual dysfunction includes sexual dysfunction (desire, arousal, orgasm, painful intercourse and vaginismus, and premature ejaculation) and paraphilias. Sexual dysfunction is seen in a significant proportion of men and women, depending on the type of disorder (3).

Attempts to identify predisposing risk factors for sleep disorders and sexual dysfunction have highlighted the role of some demographic and clinical variables (4-8). However, the unstable nature of these risk factors has not been able to help differential diagnoses of the wide range of these disorders. Therefore, it is assumed that the differential diagnosis of sleep disorders and sexual dysfunction requires screening of some relatively stable risk factors such as personality traits $(9,10)$. Recently, some studies have examined the personality correlations of these disorders based on factor analysis approaches that have found contradictory findings $(9,10)$. However, given that sleep and its disorders are still one of the least understood phenomena in biology (11), in the last two decades, the study of personality correlations of these disorders based on psychobiological approaches has accelerated (12-15).

In models based on personality psychobiological theories, temperament (and/or character) abnormalities are the main cause of psychiatric disorders $(16,17)$. The main psychobiological theories of personality include Cloninger's temperament and character model $(18,19)$, the positive and negative affect/ temperament model proposed by Watson et al. (20), Akiskal's affective temperaments model (21), and the affective and emotional composite temperament proposed by Lara et al. (22). So far, several studies have examined the relationship between the structures of these theories and sleep disorders and sexual dysfunction (11-14,23-28). However, the findings are very scattered and the integrated interpretation of them is challenging. For example, two studies $(29,30)$ have found dissimilar findings on the relationship between harm avoidance (HA) in Cloninger's theory and insomnia disorder. Other studies have also reported contradictory results on the relationship between positive affect and sleepiness $(31,32)$. On the other hand, some studies have suggested inconsistent results about the relationship between $\mathrm{HA}$ and sexual satisfaction $(33,34)$. Thus, the combined findings of previous studies in the form of a comprehensive review could help to the differential diagnosis of sleep disorders and sexual dysfunction using personality psychobiological theories. So, the present review was conducted to identify four personality psychobiological models by Cloninger $(18,19)$, Watson et al. (20), Akiskal et al. (21), and Lara et al. (22) related to sleep disorders and sexual dysfunction.

\section{Materials and Methods}

\section{Databases, Search Strategies, and Selection Criteria}

The present systematic review and meta-analysis process, including the determination of data sources and databases, search strategies, quality assessment of studies, and data extraction, follows the PRISMA 27-item checklist guidelines. Web of Science, Scopus, PubMed, ProQuest, and Cochrane were searched for English-language records published during January 1990 and April 2019. In the search strategy, first, the keywords related to the purpose of the study were extracted based on previous studies and reviews. Then, the systematic search of keywords in the title/abstract of the records was done by two members of the research team (AH \& SK). The list of keywords included ["Temperament" OR "temperament and character inventory ( $\mathrm{TCl}$ )" $\mathrm{OR}$ "TCl-R" OR "tridimensional personality questionnaire (TPQ)" OR "novelty seeking" OR "harm avoidance" OR "reward dependence" OR "persistence" OR "self-directedness" OR "cooperativeness" OR "selftranscendence" OR "affective temperament" OR "TEMPS-A" OR "positive and negative affect schedule (PANAS)" OR "positive affect" OR "negative affect" OR "affective emotional composite temperament" OR "AFECTS"] AND ["sleep-wake disorders" OR "insomnia disorder" OR "hypersomnolence disorder" OR "narcolepsy" OR "obstructive sleep apnea hypopnea" OR "central sleep apnea" OR "sleep-related hypoventilation" OR "circadian rhythm sleep-wake disorders" OR "parasomnias" OR "sleepwalking" OR "sleep terrors" OR "nightmare disorder" OR "harm avoidance" OR "restless legs syndrome" OR "sleepiness" OR "sleep quality"] (OR) ["sexual disorders" OR "sexual dysfunctions" OR "delayed ejaculation" OR "erectile disorder" OR "orgasmic disorder" OR "arousal disorder" OR "penetration disorder" OR "hypoactive sexual desire disorder" OR "premature ejaculation"].

The inclusion criteria for systematic review were: Englishlanguage original research containing adults aged 18 and over, publication in academic journals, and coverage of at least two keywords simultaneously. Exclusion criteria included studies containing qualitative methodology, unpublished dissertations and articles, studies with unrelated/unsuitable design, interventions without assessment in the baseline, reviews and meta-analysis, use of non-standard tools without validity and unstructured interviews in evaluating variables, not using TCl, TCl-R, TPQ, TEMPS, PANAS, AFECTS to measure temperament, conference abstracts or without full text, lack of access to the full text of the article, and low-quality reports according to the strengthening the Reporting of observational studies in epidemiology (STROBE). Also, in proportion to the type of sleep disorder or sexual dysfunction, correlation studies without a reported partial correlation coefficient or case-control studies without a reported mean and standard deviation were excluded from the meta-analysis. Studies containing duplicate samples were also excluded (Figure 1).

\section{Quality Assessment of Studies and Data Extraction}

The quality of the studies was assessed using a 22-item STROBE checklist used for cross-sectional, case-control, and longitudinal 


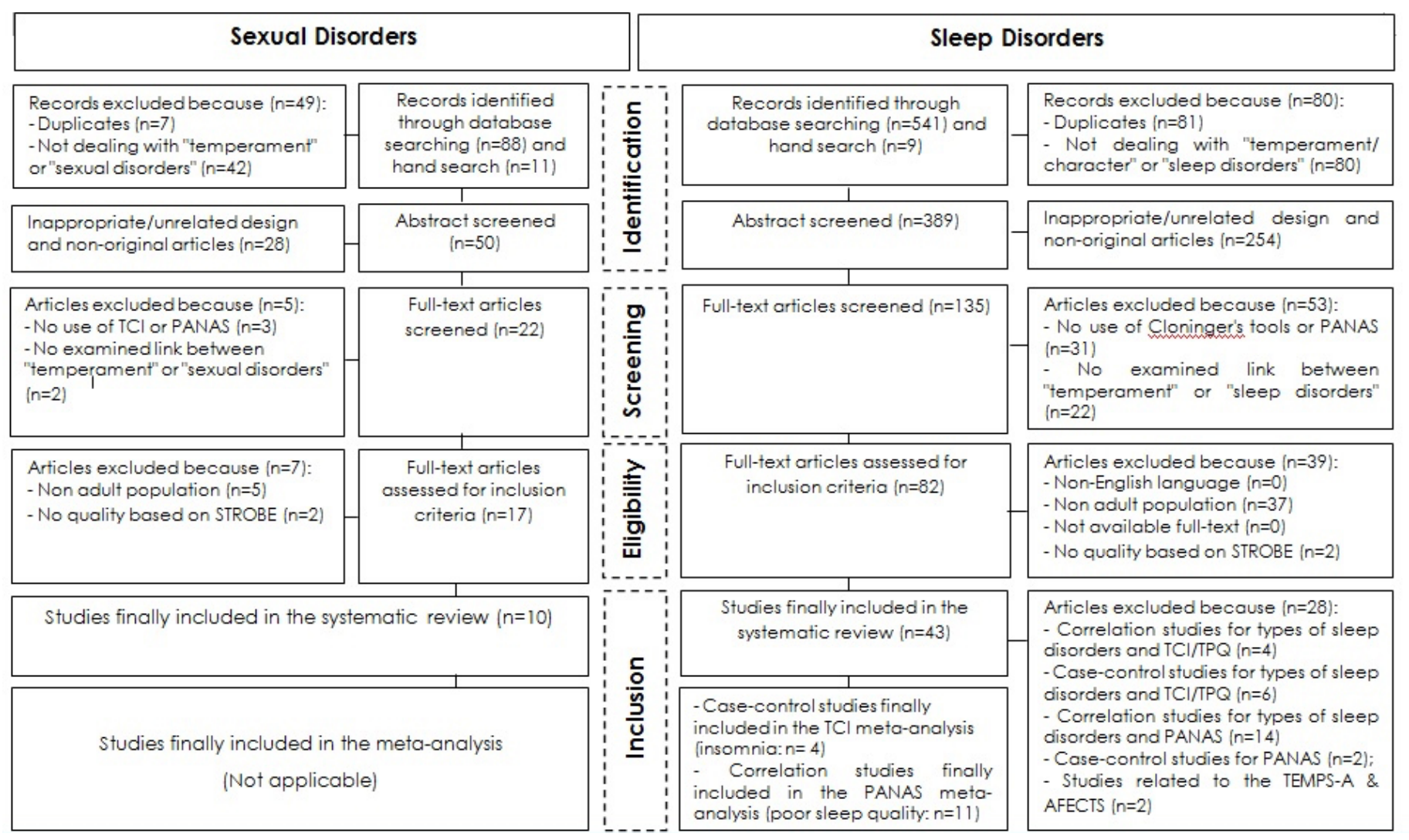

Figure 1. A flow diagram of the study selection process based on the PRISMA

studies. The process of evaluating the quality of studies was performed independently by two members of the research team (AH \& SK). The disagreement between the two assessors was resolved using the opinion of a third party (KR). Then, to extract the data, a comprehensive table was designed to record the extracted data. After selecting the appropriate quality articles, the data and information were each entered into the results registration form/table. The process of combining the extracted data was performed by the tabulation method and an accurate description of the findings of each study. The table of results was organized based on the first author and the year of study, country, design, sample size and age mean (or age range) of samples, tools, statistical analysis, related findings, and limitations and level of evidence.

\section{Statistical Analysis}

Two meta-analyses were performed to calculate (i) difference in the $\mathrm{TCl}$ subscales (novelty seeking=NS, harm avoidance=HA, reward dependence $=R D$, persistence $=P$, self-directedness $=S D$, cooperativeness $=C$, and self-transcendence $=S T$ ) between cases with insomnia and normal controls- 4 studies; and (ii) the correlation coefficients between each of the subscales of PANAS (positive affect=PA, negative affect=NA) and sleep quality assessed by the Pittsburgh Sleep Quality index-11 studies. Casecontrol studies were combined based on sample size, mean, and standard deviation of the variables in the cases and healthy controls. Pooled effects sizes for standard mean differences (cases vs. controls) are presented with $95 \%$ confidence intervals (Cl) using a combined forest plot. Cohen's d-values were used for measuring the effect size. Differences between cases and controls were compared using the Z-standard score. Correlation studies were combined based on sample sizes and partial correlation coefficients between variables. The unit of analysis was an individual study and effect sizes in these meta-analyses were obtained by the Fisher z-transformation of correlation coefficients. Estimates of the correlation coefficients in individual studies were graphically presented for each study based on the subscales of TCI or PANAS in pooled forest plots. Heterogeneity was considered in a large number of studies with $I^{2}$ higher than $50 \%$ ( $81 \%$ of studies related to sleep and $83 \%$ studies related to sexual dysfunction). Thus, pooled estimates of standard means difference was calculated for the subscales of $\mathrm{TCl}$ and types of sleep disorders using the random-effects method. Also, pooled estimates of correlation coefficients were calculated for the subscales of PANAS and sleep disorders or the subscales of $\mathrm{TCl}$ and sexual dysfunction using the randomeffects method. We studied the heterogeneity of the study samples using the $\mathrm{I}^{2}$ statistics for a $95 \% \mathrm{Cl}$. A p-value $<0.05$ for $\mathrm{I}^{2}$ higher than $50 \%$ was considered a significant heterogeneity test. Egger's test was used to possible publication bias. All hypotheses were tested at the level of $<0.05$ and performed using the comprehensive meta-analysis (CMA.2) software. 


\section{Results}

\section{Studies Included in the Systematic Review}

A systematic literature search yielded 469 papers related to sleep disorders and 99 papers related to sexual dysfunction. Finally, 43 (15 in the meta-analysis) papers were entered into the final sample related to sleep disorders, and 10 papers were entered into the final sample related to sexual dysfunction. The process of selecting and excluding studies is shown in Figure 1. Most studies have examined the relationship between the $\mathrm{TCl} /$ PANAS and sleep disorders or $\mathrm{TCl}$ and sexual dysfunction. Very few studies have been found on the relationship between the TEMPS/AFECTS and sleep disorders or sexual dysfunction. The summary of the methods and results of the studies can be seen in Tables 1 and 2.

\section{Differences Between Cases with Sleep Disorders and Controls in the Subscales of TCI}

Table 3 summarizes the systematic review results of $\mathrm{TCl}$ and sleep disorders. As can be seen in this table, the number of studies in the field of parasomnias, restless legs syndrome (RLS), and sleep breathing disorders are very limited. Differences between cases with insomnia disorder and healthy controls in the subscales of $\mathrm{TCl}$ can be seen in Figure 2. The effect sizes in the pooled forest plot with $95 \% \mathrm{Cl}$, mean and range are presented. Also, the Z-standard score and its statistical significance are presented for those subscales that have significant differences. As can be seen, $\mathrm{HA}$ is significantly higher in patients with insomnia $(z=3.346$, $\mathrm{p}=0.001$ ). In other subscales of $\mathrm{TCl}$, there was no difference between the cases and controls $(p>0.05)$. The publication bias was not seen for any of the variables ( $p>0.05$ for Egger's statistic).

\section{Correlations Between the Subscales of PANAS and Sleep Disorders}

As can be seen in Table 3, according to the results of all four available studies, NA is the strongest correlates of daytime sleepiness. Also, the relationship between NA and PA with PSQI

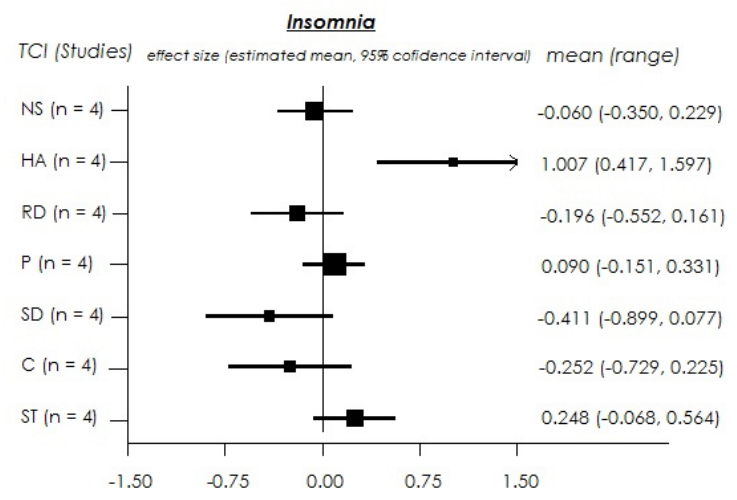

Figure 2. The pooled forest plot for the difference between cases and controls in the $\mathrm{TCl}$ subscales. Statistically significant difference for $\mathrm{HA}(\mathrm{z}=3.346, \mathrm{p}=0.001)$

TCI: Temperament and Character Inventory, HA: Harm avoidance was confirmed in $87 \%$ and $73.9 \%$ of studies, respectively. Figure 3 presents the correlations between the subscales of PANAS and sleep quality assessed by PSQI. As can be seen, there is a positive significant relationship between NA and poor sleep quality assessed by PSQI $(z=10.437, p<0.001)$. Besides, PA and poor sleep quality assessed by PSQI are negatively related $(z=-6.826, p<0.001)$. The publication bias was not seen in the relationship between any of the variables ( $p>0.05$ for Egger's statistic).

\section{Correlations Between the Subscales of $\mathrm{TCl}$ and Sexual Dysfunctions}

Table 3 shows the correlation between the subscales of $\mathrm{TCl}$ and sexual dysfunction. As can be seen, according to the results of all three available studies, $\mathrm{HA}$ and SD are the strongest correlates of sexual dissatisfaction/dysfunction. Probably, there is a positive significant relationship between $\mathrm{HA}$ and sexual dissatisfaction/dysfunction. Also, SD is likely negatively related to sexual dissatisfaction/dysfunction. Because of the limited number of available studies, the relationship between the other subscales of the $\mathrm{TCl}$ and sexual dysfunctions is ambiguous. The publication bias was not seen in the relationship between any of the variables ( $p>0.05$ for Egger's statistic).

\section{Discussion}

The study aimed to systematically review and meta-analysis the psychobiological theories of personality in sleep disorders and sexual dysfunction. The present study found that, in general, relatively few studies have examined the relationship between personality psychobiological models, particularly TEMPS and AFECTS, and sleep disorders and sexual dysfunction. However, the relationship between insomnia and $\mathrm{TCl} / \mathrm{TPQ}$, as well as the relationship between sleep quality and PANAS, has been considered in more studies. In the following, the relationship between each of these models and sleep and sexual disorders is discussed separately.

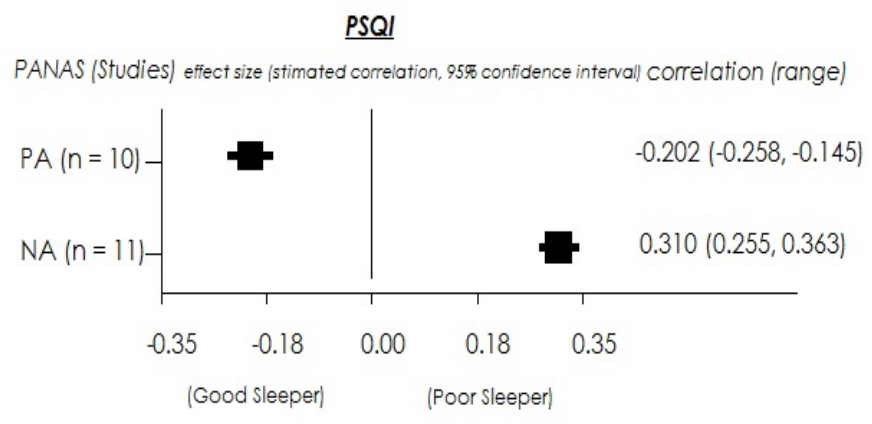

Figure 3. The pooled forest plot for correlations between PANAS subscales and sleep quality assessed using PSQI. Statistically significant correlations for PA $(z=-6.826, p<0.001)$ and NA $(z=10.437, p<0.001)$

PANAS: Positive and negative affect schedule, PSQI: Pittsburgh Sleep Quality index, PA: Positive affect, NA: Harm avoidance 


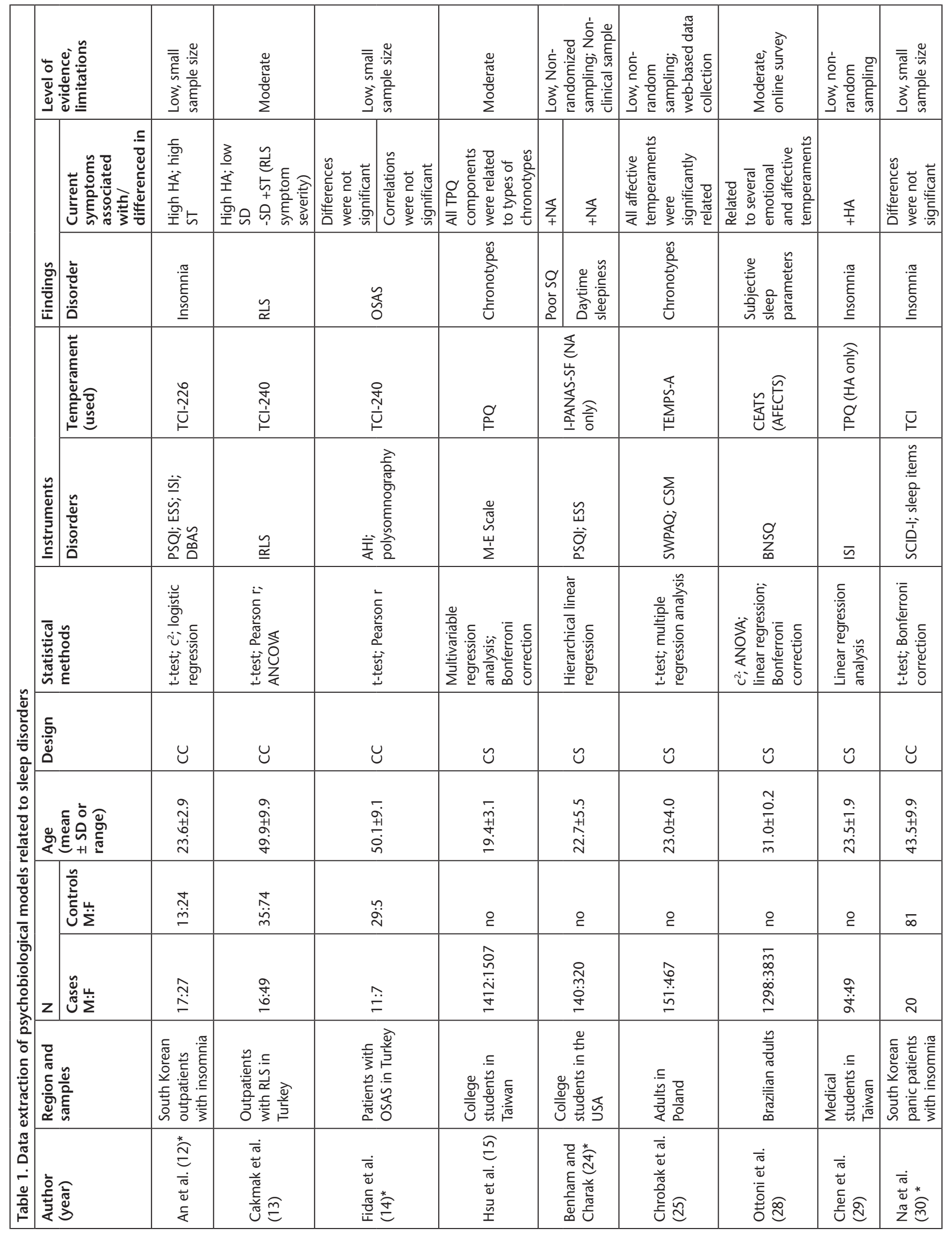




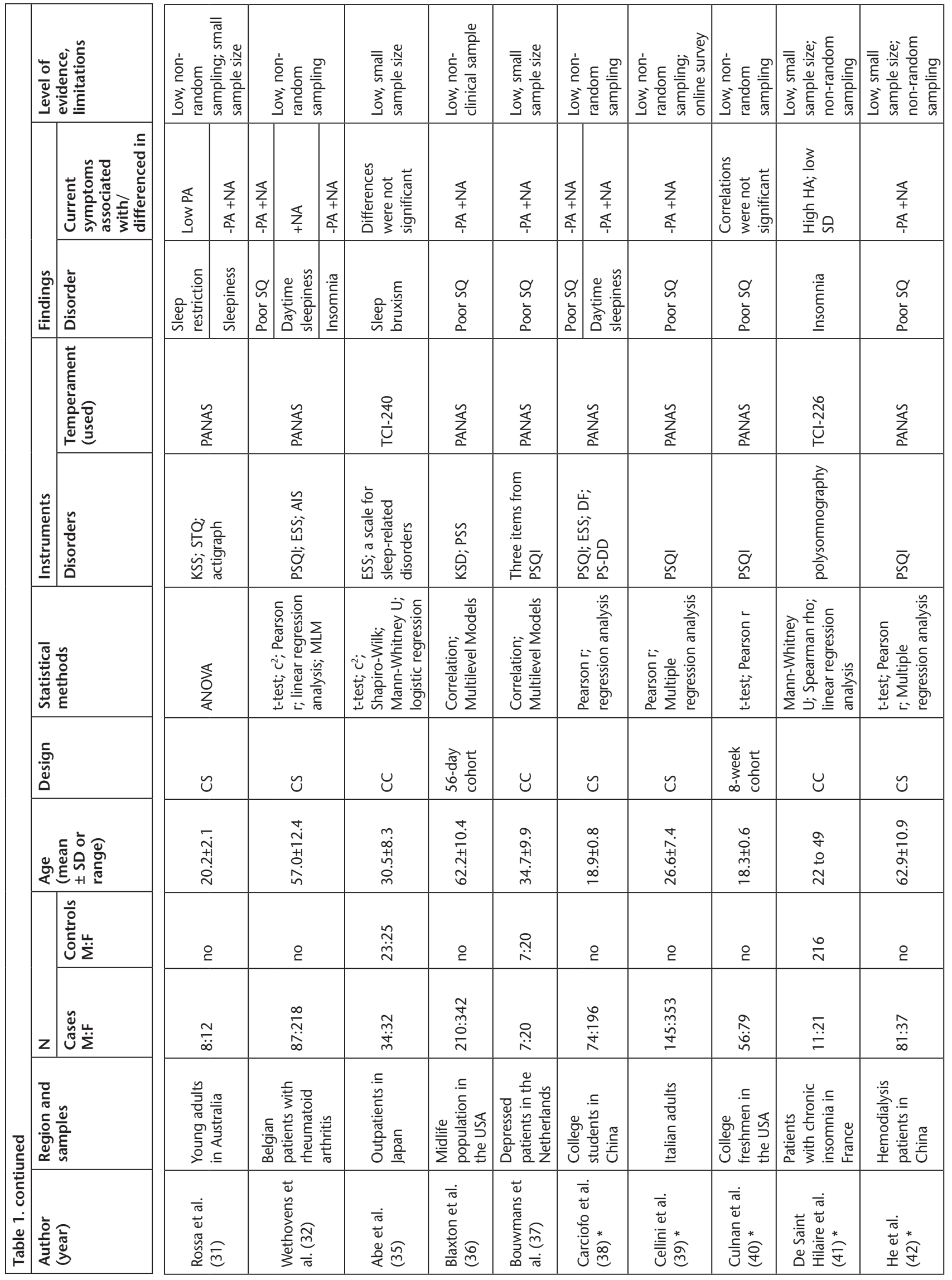


Rezaei et al.

Temperament in Sleep and Sexual Disorders

\begin{tabular}{|c|c|c|c|c|c|c|c|c|c|c|c|c|c|}
\hline \multicolumn{2}{|r|}{ 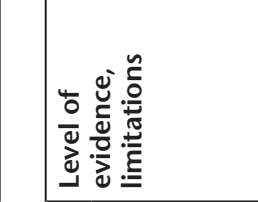 } & 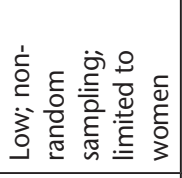 & 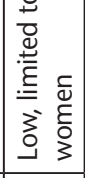 & 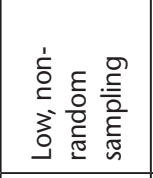 & 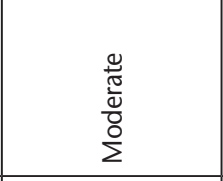 & 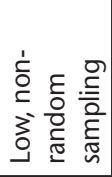 & 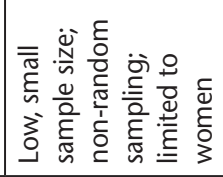 & 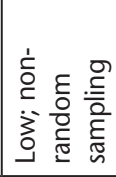 & 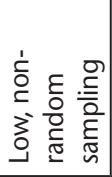 & \multicolumn{4}{|c|}{ 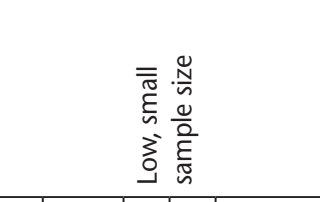 } \\
\hline & 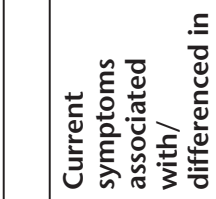 & $\sum_{+}^{\nwarrow}$ & 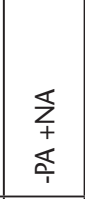 & $\begin{array}{l}\frac{1}{z} \\
+ \\
a \\
1\end{array}$ & 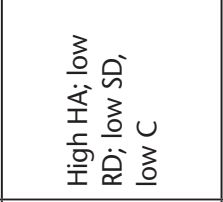 & $\lesssim$ & 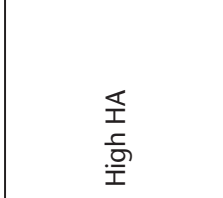 & $\underset{+}{\overleftarrow{z}}$ & $\begin{array}{l}\frac{5}{z} \\
+ \\
\vdots \\
\vdots \\
1\end{array}$ & 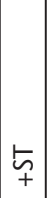 & 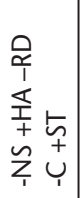 & 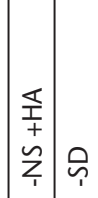 & 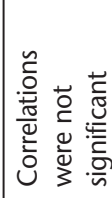 \\
\hline & 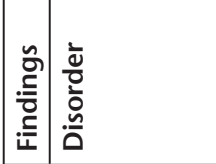 & $\begin{array}{l}\text { O } \\
\grave{o} \\
\searrow\end{array}$ & 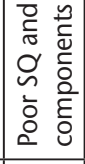 & \begin{tabular}{l}
0 \\
\hdashline \\
$\vdots$ \\
\hdashline
\end{tabular} & 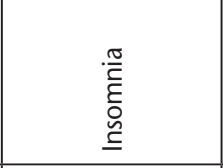 & $\begin{array}{l}0 \\
\swarrow \\
\vdots \\
\swarrow\end{array}$ & $\frac{y}{x}$ & $\begin{array}{l}\text { O } \\
\vdots \\
\vdots \\
\check{2}\end{array}$ & \begin{tabular}{l}
0 \\
$\swarrow$ \\
$\vdots$ \\
\hdashline
\end{tabular} & \begin{tabular}{c}
$\alpha$ \\
\hdashline \\
$\vdots$ \\
$\vdots$ \\
\hdashline
\end{tabular} & 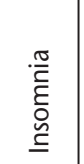 & 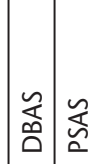 & 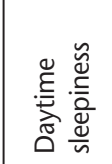 \\
\hline & 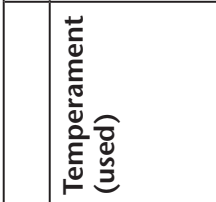 & 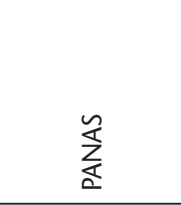 & 宏 & 否 & $\bar{\Xi}$ & 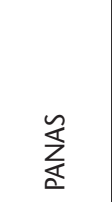 & 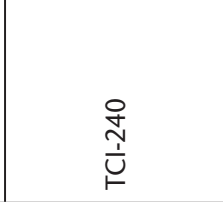 & 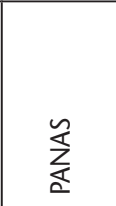 & $\sum_{\underline{\alpha}}^{n}$ & & & 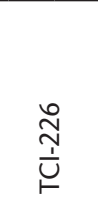 & \\
\hline & 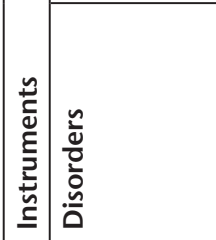 & $\bar{D}$ & $\bar{\sigma}$ & 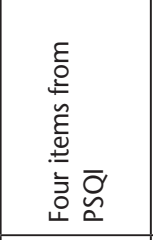 & 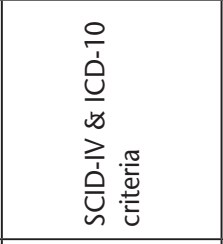 & 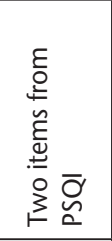 & 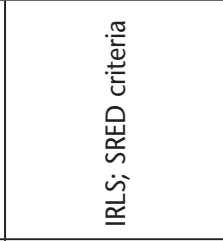 & 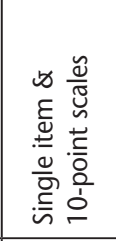 & 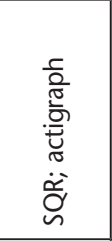 & & & 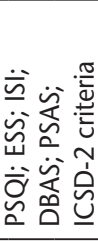 & \\
\hline & 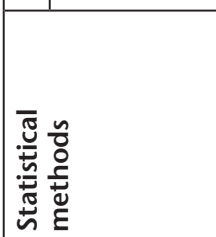 & 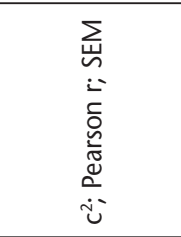 & $\sum_{\text {I }}$ & $\sum_{\text {㟧 }}$ & 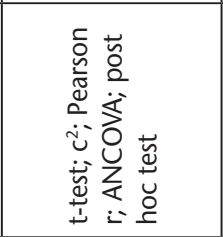 & 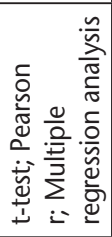 & 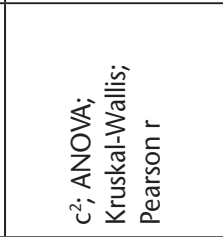 & 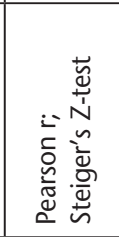 & 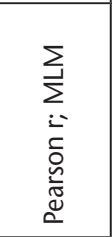 & & & 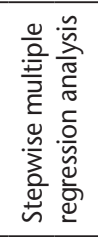 & \\
\hline & 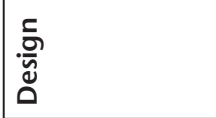 & $\tilde{u}$ & 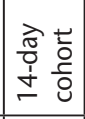 & $\begin{array}{l}\frac{\pi}{0} \\
\frac{0}{0} \\
\frac{1}{2} \\
\frac{0}{0}\end{array}$ & $\cup$ & $\tilde{u}$ & $\cup$ & $\tilde{u}$ & 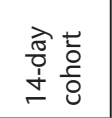 & & & $\tilde{u}$ & \\
\hline & 离六す & $\begin{array}{l}\bar{m} \\
\infty \\
\infty \\
\infty\end{array}$ & $\begin{array}{l}m \\
m \\
+1 \\
\dot{i} \\
\dot{i}\end{array}$ & $\frac{\stackrel{0}{\overline{+}}}{\frac{+1}{5}}$ & 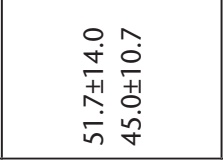 & $\begin{array}{l}\circ \\
\ddot{x} \\
+1 \\
\stackrel{N}{N}\end{array}$ & 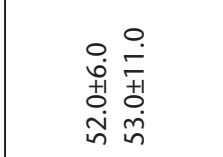 & 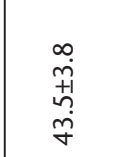 & $\begin{array}{l}\underset{i}{+} \\
\text { +1 } \\
\stackrel{N}{N}\end{array}$ & & & $\begin{array}{l}\stackrel{0}{i} \\
\stackrel{+1}{+1} \\
m \\
\stackrel{\sim}{n}\end{array}$ & \\
\hline & 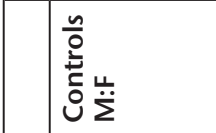 & 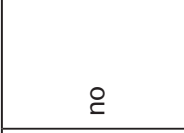 & 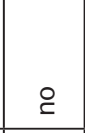 & 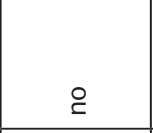 & 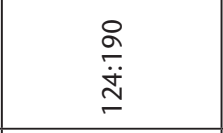 & 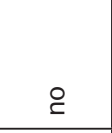 & $\stackrel{\circ}{\circ}$ & 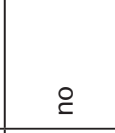 & ๕ & & & $\stackrel{\circ}{\circ}$ & \\
\hline & 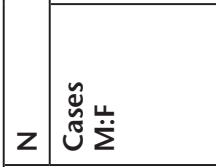 & $\begin{array}{c}\widetilde{o} \\
\tilde{0} \\
\end{array}$ & $\underset{\pi}{\pi}$ & $\frac{n}{\dddot{\ddot{\nu}}}$ & 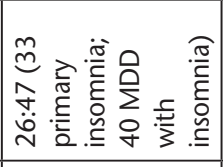 & $\begin{array}{l}\stackrel{\leftrightarrow}{0} \\
\stackrel{\otimes}{0} \\
\text { N }\end{array}$ & 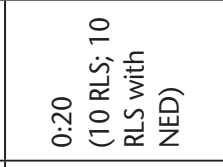 & $\begin{array}{l}R \\
\stackrel{0}{\infty} \\
\infty\end{array}$ & $\stackrel{\tilde{O}}{\circ}$ & & & 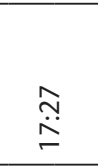 & \\
\hline 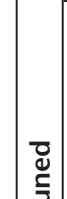 & 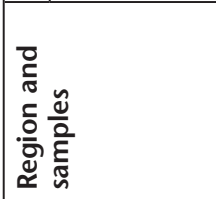 & 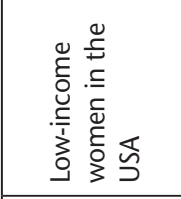 & 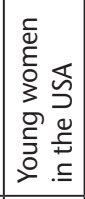 & 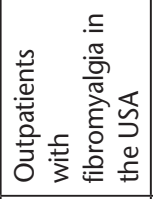 & 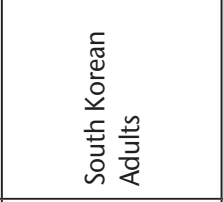 & 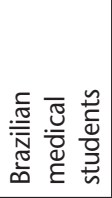 & 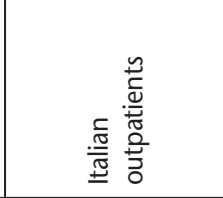 & 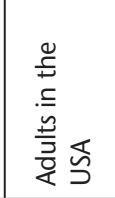 & 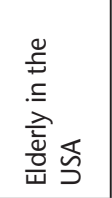 & & & 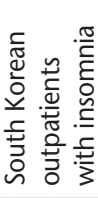 & \\
\hline 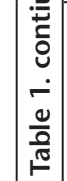 & 高 & 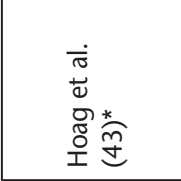 & 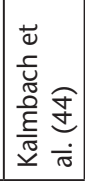 & 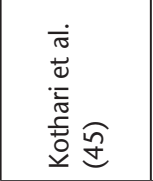 & 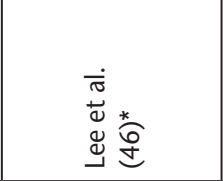 & 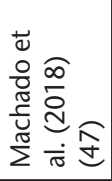 & 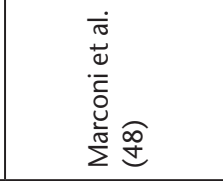 & 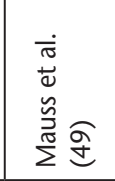 & 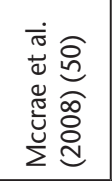 & & & 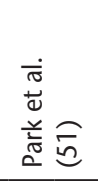 & \\
\hline
\end{tabular}


Rezaei et al.

Temperament in Sleep and Sexual Disorders

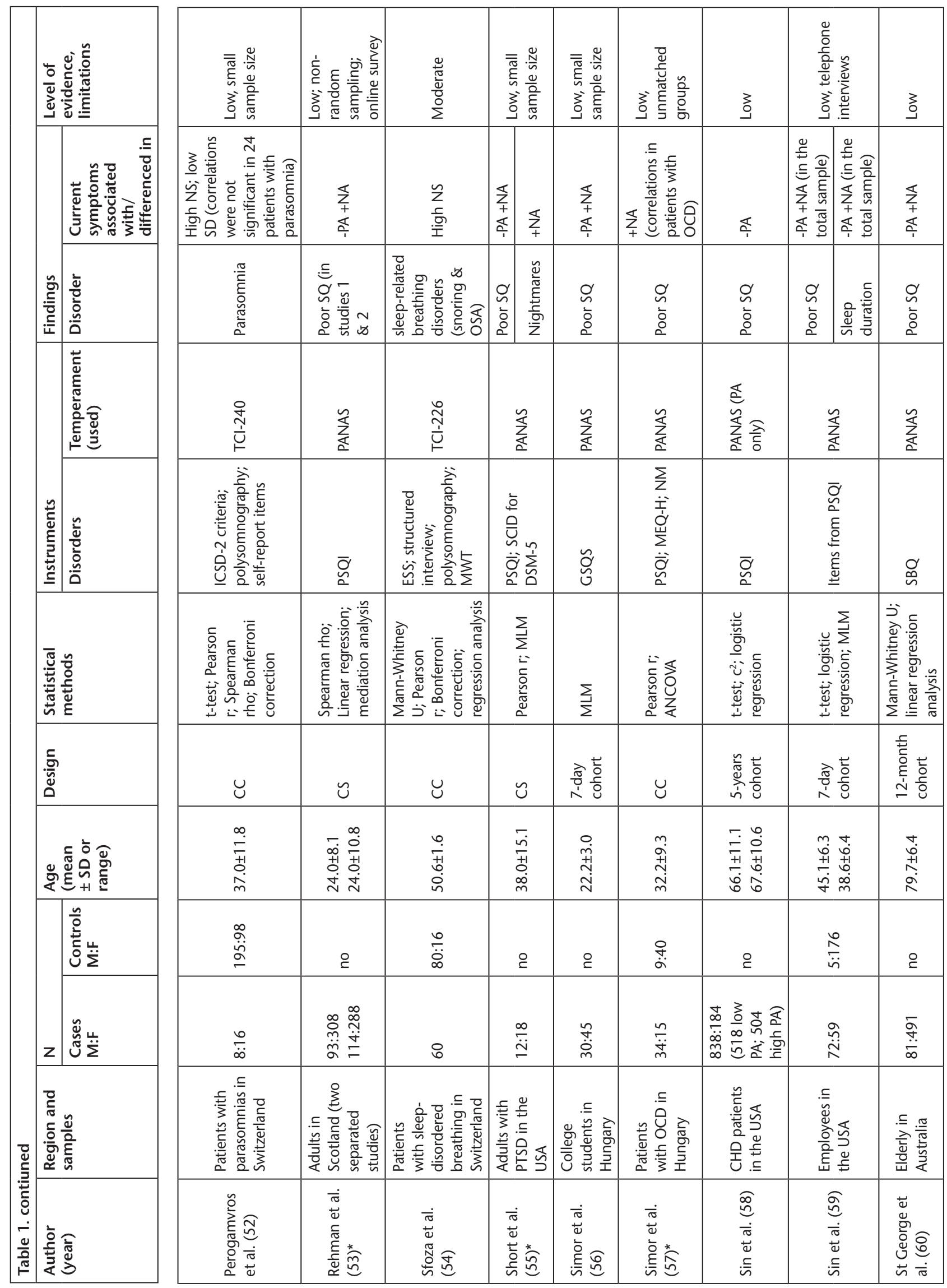


Rezaei et al

Temperament in Sleep and Sexual Disorders

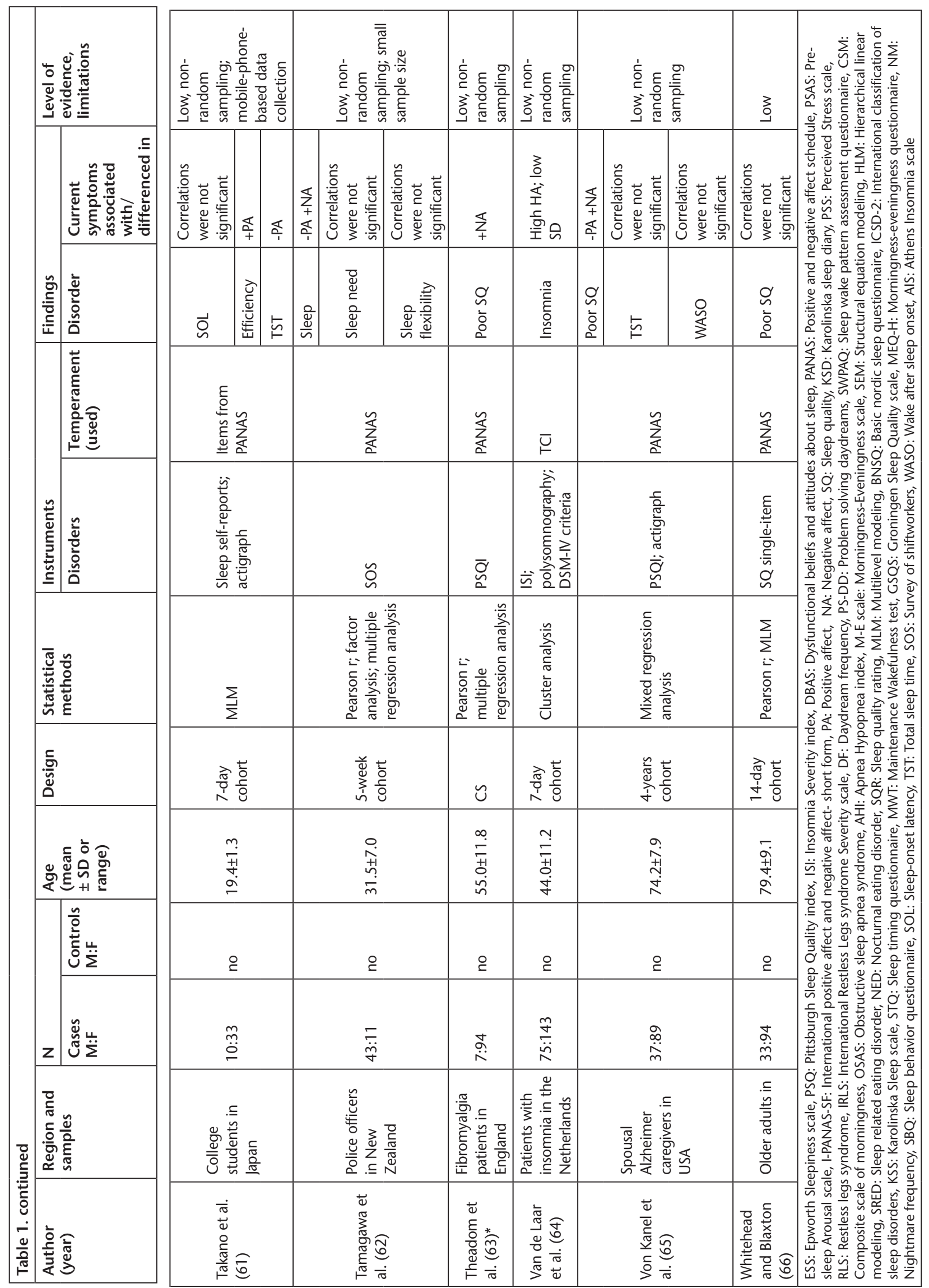


Rezaei et al.

Temperament in Sleep and Sexual Disorders

\begin{tabular}{|c|c|c|c|c|c|}
\hline 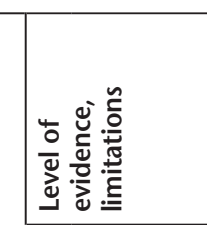 & 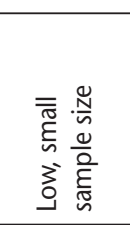 & 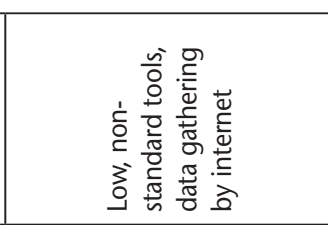 & 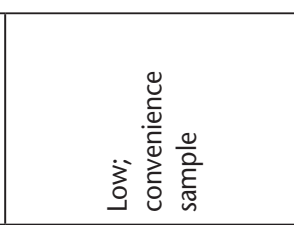 & 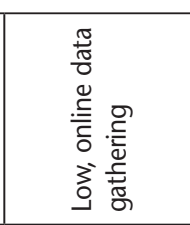 & 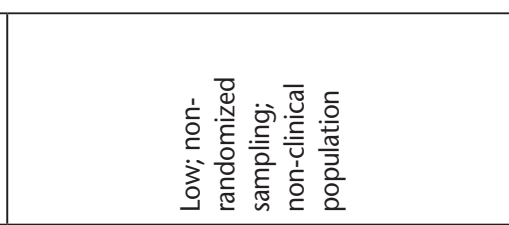 \\
\hline 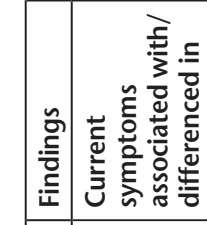 & 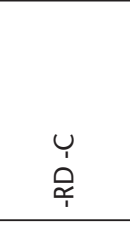 & 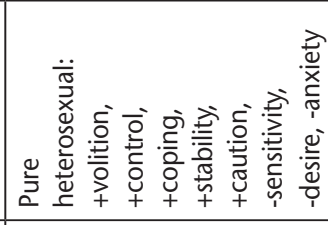 & 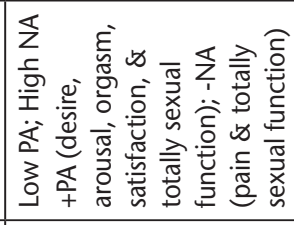 & 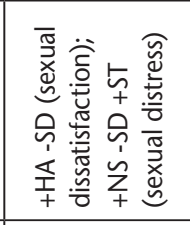 & 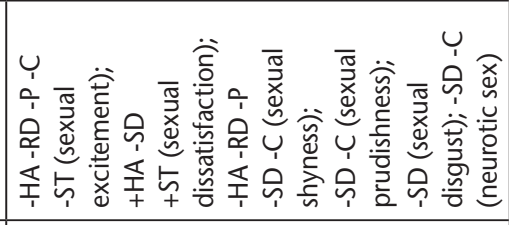 \\
\hline 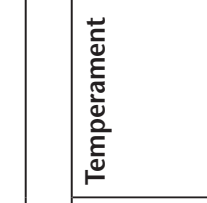 & 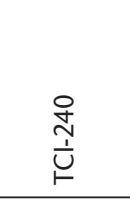 & 总 & 集 & 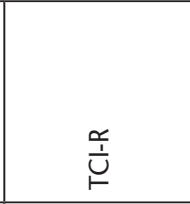 & 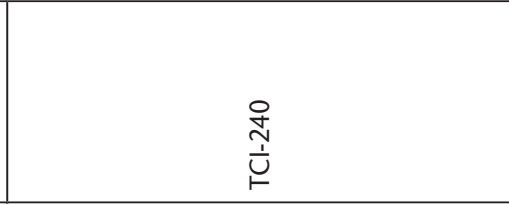 \\
\hline 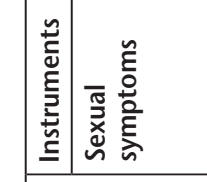 & 媔 & 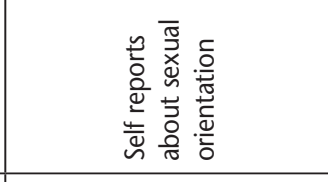 & 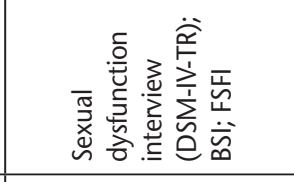 & 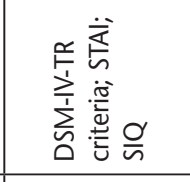 & 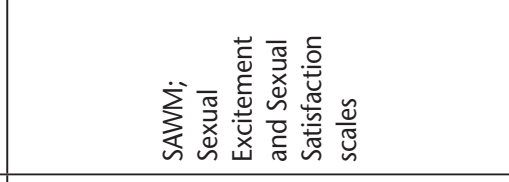 \\
\hline 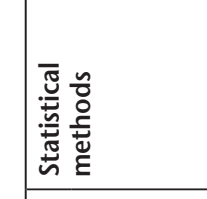 & 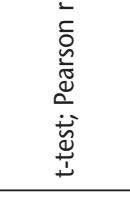 & 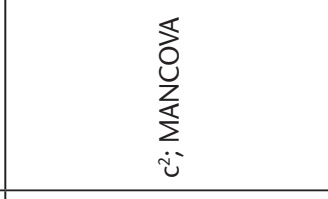 & 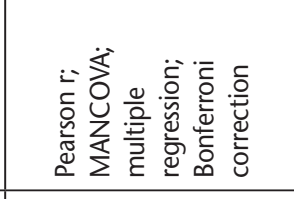 & 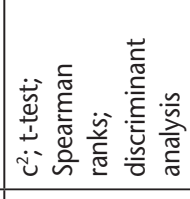 & 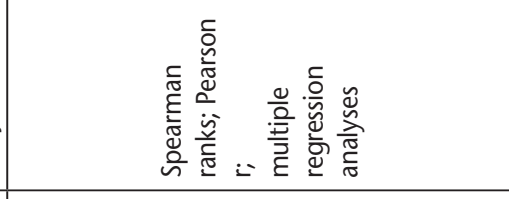 \\
\hline 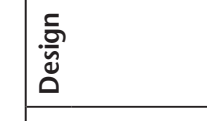 & $\ddot{u}$ & $\tilde{u}$ & u & $\tilde{u}$ & $\tilde{u}$ \\
\hline 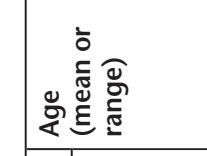 & 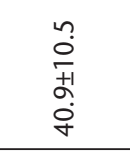 & 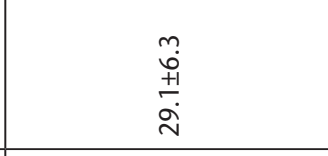 & 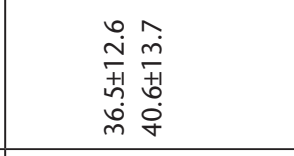 & 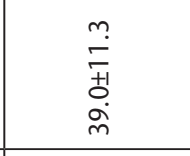 & 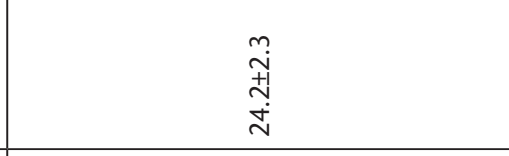 \\
\hline 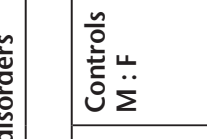 & $\stackrel{g}{\stackrel{\sigma}{\rightleftarrows}}$ & 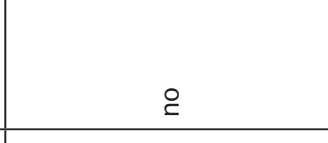 & $\begin{array}{l}\frac{\hat{\sigma}}{0} \\
\ddot{0}\end{array}$ & 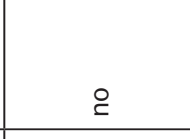 & 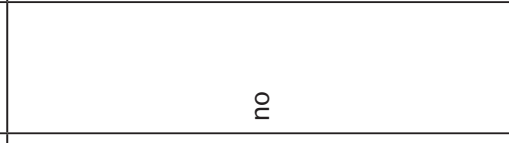 \\
\hline 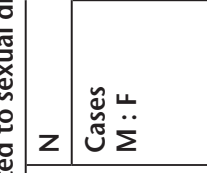 & $\stackrel{n}{n}$ & 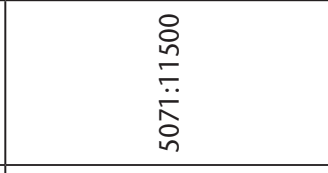 & 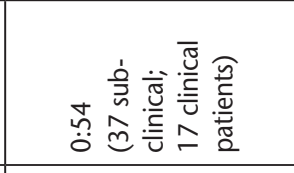 & 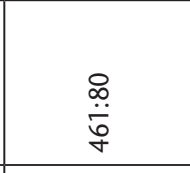 & 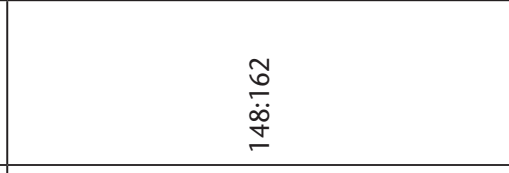 \\
\hline 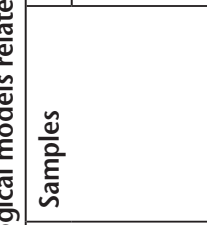 & 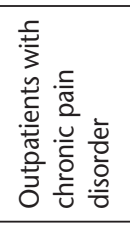 & 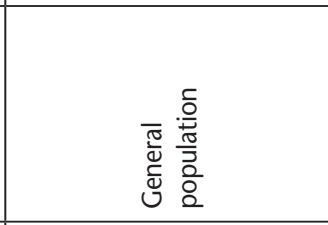 & 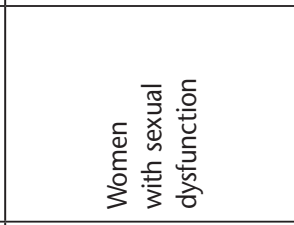 & 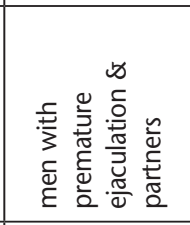 & 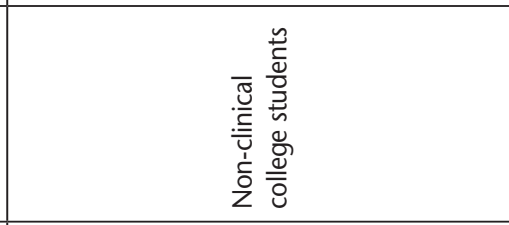 \\
\hline 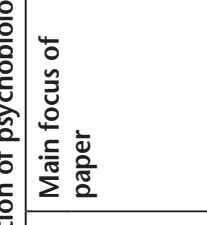 & 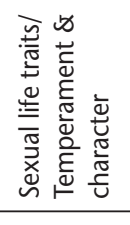 & 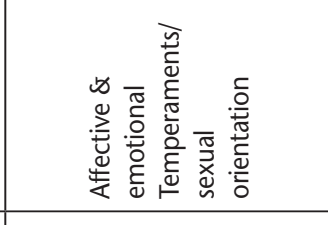 & 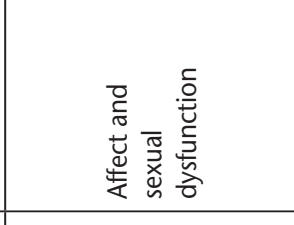 & 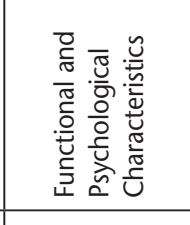 & 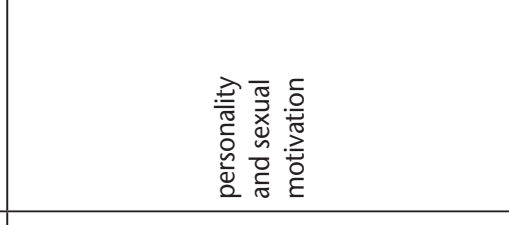 \\
\hline 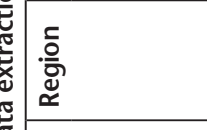 & $\begin{array}{l}\text { 离 } \\
\text { 咅 }\end{array}$ & 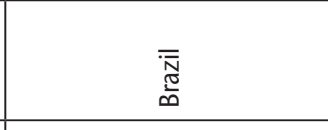 & 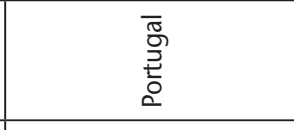 & $\begin{array}{l}\frac{\varepsilon}{5} \\
\frac{\bar{\sigma}}{\overline{0}} \\
\infty \\
\end{array}$ & 를 \\
\hline 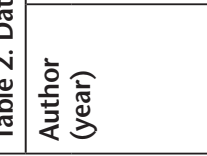 & 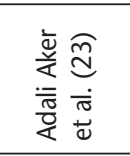 & 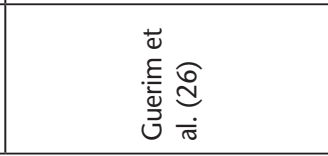 & 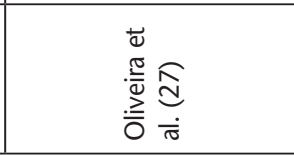 & 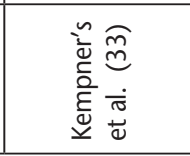 & 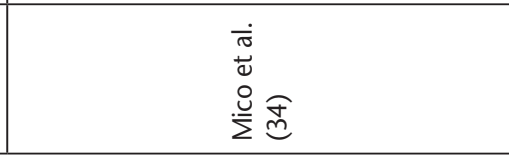 \\
\hline
\end{tabular}


Rezaei et al.

Temperament in Sleep and Sexual Disorders

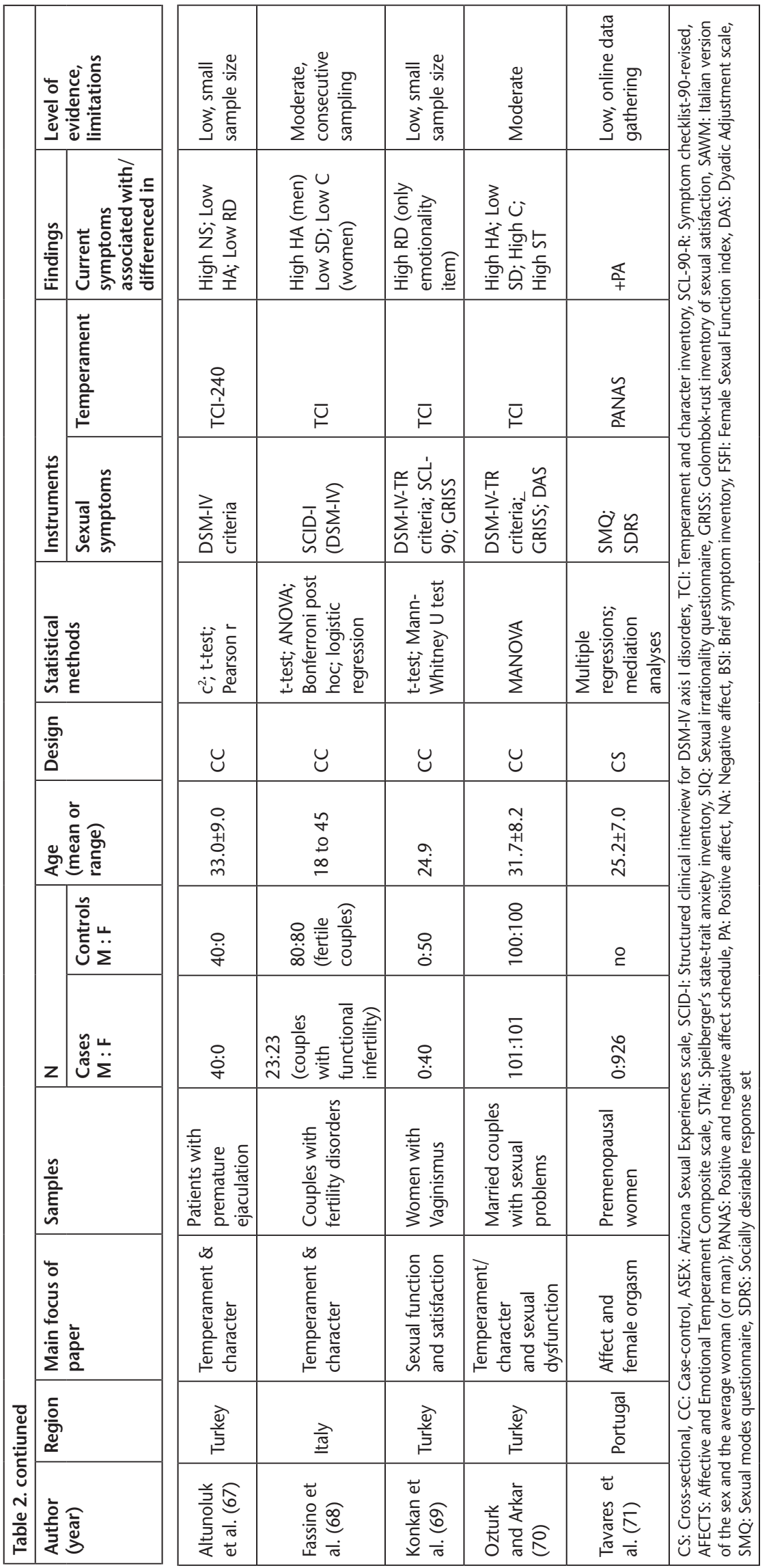

Insomnia: The results of the present metaanalysis showed that the HA in patients suffering from insomnia is higher than healthy controls. Although few studies met inclusion criteria for the meta-analysis $(12,30,41,46)$, other studies in the present review have also pointed to the relationship between these two components $(29,51)$. People with high HA have traits such as fear, embarrassment, and pessimistic worries and are easily tired, which can play a role in insomnia $(12,41)$. High HA is strongly associated with anxiety and depressive disorders $(17,72)$ and the symptoms of anxiety and depression can lead to insomnia (73). In fact, insomnia may be a marker for undiagnosed anxiety and depression (41). In addition to the above, the role of genetics and neurobiological mechanisms can also be discussed. Some components of HA, including fatigability (HA4) and anticipatory worry (HA1), are associated with reduced REM sleep duration and REM delay and these REM sleep disorders are biological markers of depression in patients with insomnia (41).

Sleep quality: Considering the very few studies related to sleep quality using $\mathrm{TCl}$, in the present meta-analysis, the relationship between PA/NA evaluated using PANAS with this component was investigated $(24,38$ $40,42,53,55,56,63)$. The current results showed that there is a positive relationship between NA and poor sleep quality. Also, PA has a negative relationship with poor sleep quality. PA and NA are the most important correlations of neuroticism traits in the fivefactor model (74). PA/NA and neuroticism are highly correlated with symptoms of anxiety and depression $(74,75)$. Other studies have suggested a link between NA components including fear, distress, guilt, anxiety, sadness, frustration, fatigue, embarrassment, and hostility with sleep problems (76-78). Therefore, it seems that poor sleep quality, which indicates poor quantity and quality of sleep, is affected by high NA and low PA. This is likely to lead to daytime sleepiness. Some studies have suggested a link between poor sleep quality and daytime sleepiness $(24,38,79)$.

Other sleep disorders: There were very few studies on parasomnias, RLS, sleep breathing disorders, and daytime sleepiness. Only two studies covered RLS $(13,48)$, both of which indicated a positive relationship between $\mathrm{HA}$ 
and RLS. Therefore, it is uncertain whether high HA is an important component in RLS. Two studies also have noted a significant relationship between low SD and RLS/parasomnias $(13,52)$. Two studies reported a significant relationship between NS and parasomnias/sleep breathing disorders $(52,54)$. On the other hand, several studies have suggested a significant relationship between NA/PA and parasomnias/daytime sleepiness $(24,31,32,38,55)$. Despite the reported relationships between psychobiological models and sleep disorders in the recent section, achieving more accurate results requires metaanalysis on a large number of studies.

Sexual dysfunction: Few studies in this area, as well as the wide range of types of sexual dysfunction, made it difficult to conduct an integrated analysis. Previous studies have sporadically examined the role of personality components in sexual dysfunction/dissatisfaction $(33,34,70)$, sexual distress (33), sexual experiences and arousal $(23,34)$, and premature ejaculation (67). Only three studies covered sexual dysfunction/ dissatisfaction, all of which indicated a positive relationship between this component and HA $(33,34,70)$. Also, all three studies reported a significant relationship between sexual dysfunction/dissatisfaction and SD. Therefore, based on the results of the current review, there is an uncertain possibility that HA and SD are two effective components in dysfunction/ sexual dissatisfaction. Despite the complexity of the issue, higher levels of HA indicate anxious tendencies, and may this component is a causal factor for sexual dysfunction through a type of serotonergic deficiency $(33,34)$. On the other hand, Cloninger introduces the character as conscious consciousness programs that consist of three individual (SD), social (C), and global (ST) cognitive sets. Unlike the temperaments that are considered as habitual systems and unconscious automatic reactions, characters are the mental and cognitive dimensions that provide a conceptual insight for the individual (80). Disorder in the characters, especially SD, can lead to the development and persistence of mental disorders $(81,82)$.

\section{Study Limitations}

Current systematic review and meta-analysis in the field of the relationship between personality psychobiological theories and sleep disorders/sexual dysfunction is a pioneering study. However, there were some limitations. The lack of numerous studies related to TEMPS and AFECTS has prevented us from doing meta-analyses and any summary and scientific conclusion. This limitation should not lead to the neglect of the suggested models by Akiskal et al. (21) and Lara et al. (22) in future studies. Therefore, if researchers have access to studies related to these models, future reviews and meta-analyses can cover them. A small number of studies related to TCl/TPQ and insomnia and examined small sample sizes can be effective in reducing the validity of the results. The wide range of types of sexual dysfunction and related factors such as dissatisfaction, distress, embarrassment, disgust, and sexual arousal also made it difficult to draw an accurate comprehensive conclusion. This heterogeneity between variables and the lack of case-control studies focused exclusively on one special disorder can also be a serious challenge to generalizing the findings to a variety of sexual disorders. If more studies are available in the next decade, analyzes can also take into account age and sex differences. Participants in the studies that were entered into the metaanalysis of sleep quality assessed PSQI were a combination of the clinical and general population. Although PSQI can be used as a dimensional instrument (without a cut-off point), the use of case-control studies containing established poor sleepers can provide more accurate results. Another limitation is related to psychiatric comorbidities with sleep disorders, which were not rule-out in the present meta-analyses. Therefore, repetition of the present study in the coming years, if there are enough casecontrol studies for meta-analysis, can provide more accurate and valid findings for specialists and clinicians.

\section{Conclusions}

Studies of sleep disorders and sexual dysfunctions based on personality psychobiological models, especially case-control studies, are very few. Although, the bigger challenge is the wide range of these disorders (insomnia, sleep breathing disorders and its sub-components, central hypersomnolence disorders, parasomnias, sleep-related movement disorders, circadian rhythm sleep-wake disorders, poor sleep quality, and sexual disorders related to the desire, arousal, orgasm, painful intercourse and vaginismus, premature ejaculation, and paraphilias) that make it difficult to draw a comprehensive meta-analysis and conclusion. The affective and emotional temperaments assessed by TEMPS-A and AFECTS have largely been ignored. Considering the importance of biomarkers in the pharmacotherapy of sleep/sexual disorders, future studies are encouraged to examine the relationship between the two models and the diagnosis of these disorders.

The models measured using $\mathrm{TCl}$ and PANAS, especially concerning sleep disorders, have produced relatively more studies. According to the findings of the present meta-analysis, NA/PA and HA may play a key role in sleep quality and insomnia, respectively. At lower levels of confidence from the current review, HA and SD along with NA may be associated with other sleep disorders. Also, HA and SD are likely to be effective components in sexual dissatisfaction/dysfunction.

Although there was no publication bias related to the sleep/ sexual disorders in the meta-analyses for insomnia and sleep quality, low evidence levels were seen in approximately $90 \%$ of sleep and $80 \%$ sexual dysfunction studies. Small sample size, non-randomized sampling, and internet-telephone based assessment were among the main limitations of the studies included in this review. Therefore, although the temperament traits assessed using the $\mathrm{TCl}$ and PANAS can potentially be important, to obtain findings with more acceptable validity, a future meta-analysis may provide valuable findings if access to a greater number of case-control studies and a focus on age and sex differences. 
Rezaei et al.

Temperament in Sleep and Sexual Disorders

\begin{tabular}{|c|c|c|c|c|c|c|c|}
\hline $\begin{array}{l}\text { Sleep/sexual } \\
\text { disorders }\end{array}$ & $\begin{array}{l}\text { Proportion } \\
\text { of reports } \\
\text { (\%) }\end{array}$ & $\begin{array}{l}\text { Association of } \\
\text { the disorder and } \\
\text { temperament } \\
\text { (positive } \\
\text { relation/ higher } \\
\text { if the presence of } \\
\text { disorder) }\end{array}$ & References & $\begin{array}{l}\text { Proportion of } \\
\text { reports (\%) }\end{array}$ & $\begin{array}{l}\text { Association of } \\
\text { the disorder and } \\
\text { temperament } \\
\text { (negative } \\
\text { relation/lower if } \\
\text { the presence of } \\
\text { disorder) }\end{array}$ & References & $\begin{array}{l}\text { Number } \\
\text { of studies } \\
\text { qualified for } \\
\text { a meta- } \\
\text { analysis }\end{array}$ \\
\hline \multirow{8}{*}{ Insomnia } & & NS & & $1 / 7(14.3)$ & NS & 51 & $4^{*}$ \\
\hline & 6/7 (85.7) & $\mathrm{HA}$ & $\begin{array}{l}12,29,41,46, \\
51,64\end{array}$ & & $\mathrm{HA}$ & & $4^{*}$ \\
\hline & & $\mathrm{RD}$ & & $2 / 7(28.6)$ & $\mathrm{RD}$ & 46,51 & $4^{*}$ \\
\hline & & SD & & $3 / 7(42.6)$ & SD & $41,46,64$ & $4^{*}$ \\
\hline & & $\mathrm{C}$ & & $2 / 7(28.6)$ & $\mathrm{C}$ & 46,51 & $4^{*}$ \\
\hline & $2 / 7$ (28.6) & ST & 12,51 & & ST & & $4^{*}$ \\
\hline & & PA & & $1 / 1$ & PA & 32 & 1 \\
\hline & $1 / 1$ & NA & 32 & & NA & & 1 \\
\hline \multirow{3}{*}{ Parasomnias } & $1 / 2(50)$ & NS & 52 & & NS & & 2 \\
\hline & & SD & & $1 / 2(50)$ & SD & 52 & 2 \\
\hline & 1/1 (100) & NA & 55 & & NA & & 1 \\
\hline \multirow{4}{*}{$\begin{array}{l}\text { Restless legs } \\
\text { syndrome (RLS) }\end{array}$} & & NS & & & NS & & 2 \\
\hline & $2 / 2(100)$ & $\mathrm{HA}$ & 13,48 & & $\mathrm{HA}$ & & 2 \\
\hline & & SD & & $1 / 2(50)$ & SD & 14 & 2 \\
\hline & $1 / 2(50)$ & ST & 13 & & ST & & 2 \\
\hline $\begin{array}{l}\text { Sleep breathing } \\
\text { disorder (SBD) }\end{array}$ & $1 / 2(50)$ & NS & 54 & & NS & & 2 \\
\hline \multirow{3}{*}{$\begin{array}{l}\text { Pittsburgh } \\
\text { Sleep Quality } \\
\text { index (PSQI) }\end{array}$} & 1/1 (100) & ST & 51 & & ST & & 1 \\
\hline & & PA & & $17 / 23(73.9)$ & PA & $\begin{array}{l}32,36-39,42 \\
44,45,47,50,53 \\
55,57-60,65\end{array}$ & $10^{*}$ \\
\hline & 20/23 (87) & NA & $\begin{array}{l}24,32,36-39,42- \\
45,49,50,53,55- \\
58,60,63,65\end{array}$ & & NA & & $11^{*}$ \\
\hline \multirow{2}{*}{ Sleepiness } & & PA & & $2 / 3(66.7)$ & PA & 38,31 & 2 \\
\hline & $4 / 4(100)$ & NA & $24,38,31,32$ & & NA & & 3 \\
\hline \multirow{4}{*}{$\begin{array}{l}\text { Sexual } \\
\text { dysfunction/ } \\
\text { dissatisfaction }\end{array}$} & $3 / 3(100)$ & $\mathrm{HA}$ & $33,34,70$ & & $\mathrm{HA}$ & & 2 \\
\hline & & SD & & $3 / 3(100)$ & SD & $33,34,70$ & 2 \\
\hline & & C & & & C & & 2 \\
\hline & $1 / 3(33.3)$ & ST & 70 & & ST & & 2 \\
\hline \multirow{3}{*}{ Sexual distress } & 1/1 (100) & NS & 33 & & NS & & 1 \\
\hline & & SD & & $1 / 1(100)$ & SD & 33 & 1 \\
\hline & $1 / 1(100)$ & ST & 33 & & ST & & 1 \\
\hline \multirow{5}{*}{$\begin{array}{l}\text { Sexual } \\
\text { excitement }\end{array}$} & & $\mathrm{HA}$ & & $1 / 1(100)$ & $\mathrm{HA}$ & 34 & 1 \\
\hline & & $\mathrm{RD}$ & & $1 / 1(100)$ & RD & 34 & 1 \\
\hline & & $\mathrm{P}$ & & $1 / 1(100)$ & $\mathrm{P}$ & 34 & 1 \\
\hline & & C & & $1 / 1(100)$ & C & 34 & 1 \\
\hline & & ST & & $1 / 1(100)$ & ST & 34 & 1 \\
\hline \multirow{3}{*}{$\begin{array}{l}\text { Premature } \\
\text { ejaculation }\end{array}$} & $1 / 1(100)$ & NS & 67 & & NS & & 1 \\
\hline & & $\mathrm{HA}$ & & $1 / 1(100)$ & $\mathrm{HA}$ & 67 & 1 \\
\hline & & $\mathrm{RD}$ & & 1/1 (100) & $\mathrm{RD}$ & 67 & 1 \\
\hline \multirow{2}{*}{$\begin{array}{l}\text { Sexual } \\
\text { experiences }\end{array}$} & & $\mathrm{RD}$ & & $1 / 1(100)$ & RD & 23 & 1 \\
\hline & & C & & $1 / 1(100)$ & $\mathrm{C}$ & 23 & 1 \\
\hline
\end{tabular}




\section{Acknowledgments}

This article is part of a Ph.D. thesis of Mr. Saeid Komasi entitled "Review and reconsideration of Hierarchical Taxonomy of Psychopathology (HiTOP) model with an emphasis on psychobiological models in the explanation of somatoform disorders", financial support and sponsored by the Neurosciences Research Center and the Vice-Chancellor for Research \& Technology of Kurdistan University of Medical Sciences, Sanandaj, Iran (ID: 98724).

\section{Ethics}

The project was approved and received a code of ethics by the Kurdistan University of Medical Sciences, Sanandaj, Iran (IR. MUK.REC.1398.169).

Peer-review: Internally peer-reviewed.

\section{Authorship Contributions}

Concept: F.R., A.H., K.R., S.K., Design: F.R., A.H., K.R., S.K., Data Collection or Processing: F.R., A.H., K.R., S.K., Analysis or Interpretation: F.R., A.H., K.R., S.K., Literature Search: F.R., A.H., K.R., S.K., Writing: F.R., A.H., K.R., S.K.

Conflict of Interest: The authors are not aware of any conflict of interest.

Financial Disclosure: The project was funded by the Kurdistan University of Medical Sciences, Sanandaj, Iran (ID: 98724).

\section{References}

1. Cho JW, Duffy JF. Sleep, sleep disorders, and sexual dysfunction. World J Mens Health 2019;37:261-75.

2. Khazaie $\mathrm{H}$, Komasi $\mathrm{S}$. The importance of screening sleep disorders in outpatient cardiac rehabilitation programs in Iran. ARYA Atheroscler 2018; 14:145-6.

3. McCabe MP, Sharlip ID, Lewis R, Atalla E, Balon R, Fisher AD, Laumann E, Lee SW, Segraves RT. Incidence and prevalence of sexual dysfunction in women and men: A consensus statement from the Fourth International Consultation on Sexual Medicine 2015. J Sex Med 2016;13:144-52.

4. Esposito G, Odelli V, Romiti L, Chiaffarino F. Prevalence and risk factors for restless legs syndrome during pregnancy in a Northern Italian population. J Obstet Gynaecol 2019;39:480-4.

5. McCabe MP, Sharlip ID, Lewis R, Atalla E, Balon R, Fisher AD, Laumann E, Lee SW, Segraves RT. Risk factors for sexual dysfunction among women and men: A consensus statement from the Fourth International Consultation on Sexual Medicine 2015. J Sex Med 2016;13:153-67.

6. McCool-Myers M, Theurich M, Zuelke A, Knuettel H, Apfelbacher C. Predictors of female sexual dysfunction: a systematic review and qualitative analysis through gender inequality paradigms. BMC Womens Health 2018;18:108.

7. Smagula SF, Stone KL, Fabio A, Cauley JA. Risk factors for sleep disturbances in older adults: evidence from prospective studies. Sleep Med Rev 2016;25:21-30.

8. van der Spuy I, Zhao G, Karunanayake C, Pahwa P. Predictors of sleep apnea in the Canadian population. Can Respir J 2018;2018:6349790.

9. Hintsanen M, Puttonen S, Smith K, Tornroos M, Jokela M, PulkkiRaback L, Hintsa T, Merjonen P, Dwyer T, Raitakari OT, Venn A, Keltikangas-Järvinen L. Five-factor personality traits and sleep: evidence from two population-based cohort studies. Health Psychol 2014;33:1214-23.
10. Stephan Y, Sutin AR, Bayard MS, Križan Z, Terracciano A. Personality and sleep quality: evidence from four prospective studies. Health Psychol 2018;37:271-81.

11. Sehgal A, Mignot E. Genetics of sleep and sleep disorders. Cell 2011;146:194-207.

12. An H, Park J, Jang ES, Chung S. The impact of temperament and character on the efficacy of nonpharmacologic treatment of primary insomnia. Compr Psychiatry 2012;53:201-7.

13. Cakmak VA, Gazioglu S, Usta NC, Ozkorumak E, Ayar A, Topbas M, Boz C. Evaluation of Temperament and character features as risk factors for depressive symptoms in patients with restless legs syndrome. J Clin Neurol 2014;10:320-7.

14. Fidan F, Özbulut Ö, Aycicek A, Asik AH, Ünlu M. Effects of obstructive sleep apnea syndrome on temperament and character. Turk J Med Sci 2013;43:138-43.

15. Hsu CY, Gau SSF, Shang CY, Chiu YN, Lee M-B. Associations between chronotypes, psychopathology, and personality among incoming college students. Chronobiol Int. 2012;29:491-501.

16. Kampman O, Viikki M, Järventausta K, Leinonen E. Meta-analysis of anxiety disorders and temperament. Neuropsychobiology 2014;69:175-86.

17. Miettunen J, Raevuori A. A meta-analysis of temperament in axis psychiatric disorders. Compr Psychiatry 2012;53:152-66.

18. Cloninger CR. A systematic method for clinical description and classification of personality variants. Arch Gen Psychiatry 1987;44:573-88.

19. Cloninger CR, Svrakic DM, Przybeck TR. A psychobiological model of temperament and character. Arch Gen Psychiatry 1993;50:975-90.

20. Watson D, Clark LA, Tellegen A. Development and validation of brief measures of positive and negative affect: the PANAS scales. J Pers Soc Psychol 1988;54:1063-70.

21. Akiskal HS, Djenderedjian AM, Rosenthal RH, Khani MK. Cyclothymic disorder: validating criteria for inclusion in the bipolar affective group. Am J Psychiatry 1977;134:1227-33.

22. Lara DR, Bisol LW, Brunstein MG, Reppold CT, de Carvalho HW, Ottoni GL. The Affective and Emotional Composite Temperament (AFECT) model and scale: a system-based integrative approach. J Affect Disord 2012;140:14-37.

23. Adali Aker D, Solmaz M, Kose S. Sexual life traits of chronic pain disorder patients and relationship between temperament and character dimensions of personality. Psychiatry Behav Sci 2019;9:23-33.

24. Benham G, Charak R. Stress and sleep remain significant predictors of health after controlling for negative affect. Stress Health 2019;35:59-68.

25. Chrobak AA, Tereszko A, Dembinska-Krajewska D, Arciszewska A, Siwek M, Dudek D, Rybakowski J. Morningness-eveningness and affective temperaments assessed by the Temperament Evaluation of Memphis, Pisa and San Diego-Autoquestionnaire (TEMPS-A). Chronobiol Int 2017;34:57-65.

26. Guerim DL, De Carvalho H, Lara D. The relationship between temperament and sexual orientation. 2015;175:379-84.

27. Oliveira C, Nobre PJ. The role of trait-affect, depression, and anxiety in women with sexual dysfunction: a pilot study. J Sex Marital Ther 2013;39:436-52.

28. Ottoni GL, Lorenzi TM, Lara DR. Association of temperament with subjective sleep patterns. J Affect Disord 2011;128:120-7.

29. Chen CY, Yu NW, Huang TH, Wang WS, Fang JT. Harm avoidance and depression, anxiety, insomnia, and migraine in fifth-year medical students in Taiwan. Neuropsychiatric disease and treatment 2018;14:1273-80 
30. Na HR, Kang EH, Yu BH, Woo JM, Kim YR, Lee SY, Chung SK. Relationship between personality and insomnia in panic disorder patients. Psychiatry Investig 2011;8:102-6.

31. Rossa KR, Smith SS, Allan AC, Sullivan KA. The effects of sleep restriction on executive inhibitory control and affect in young adults. J Adolesc Health 2014;55:287-92.

32. Westhovens R, Van der Elst K, Matthys A, Tran M, Gilloteau I. Sleep problems in patients with rheumatoid arthritis. J Rheumatol 2014;41:31-40.

33. Kempeneers P, Andrianne R, Bauwens S, Georis I, Pairoux JF, Blairy $S$. Functional and psychological characteristics of belgian men with premature ejaculation and their partners. Arch Sex Behav 2013;42:51-66.

34. Mico U, Scimeca G, Bruno A, Pandolfo G, Romeo VM, Mallamace D, Zoccali R, Muscatello MRA. The relationship between personality and sexual motivation: an investigation based on Cloninger's model in nonclinical Italian subjects. Riv Psichiatr 2013;48:307-14.

35. Abe Y, Suganuma T, Ishii M, Yamamoto G, Gunji T, Clark GT, Tachikawa T, Kiuchi Y, Igarashi Y, Baba K. Association of genetic, psychological and behavioral factors with sleep bruxism in a Japanese population. J Sleep Res 2012;21:289-96.

36. Blaxton JM, Bergeman CS, Whitehead BR, Braun ME, Payne JD. Relationships among nightly sleep quality, daily stress, and daily affect. J Gerontol B Psychol Sci Soc Sci 2017;72:363-72.

37. Bouwmans MEJ, Bos EH, Hoenders HJR, Oldehinkel AJ, de Jonge P. Sleep quality predicts positive and negative affect but not vice versa. An electronic diary study in depressed and healthy individuals. J Affect Disord 2017;207:260-7.

38. Carciofo R, Du F, Song N, Zhang K. Mind wandering, sleep quality, affect and chronotype: an exploratory study. PLoS One 2014;9:e91285. doi: 10.1371/journal.pone.0091285.

39. Cellini N, Duggan KA, Sarlo M. Perceived sleep quality: The interplay of neuroticism, affect, and hyperarousal. Sleep Health 2017;3:184-9.

40. Culnan E, Kloss JD, Grandner M. A prospective study of weight gain associated with chronotype among college freshmen. Chronobiol Int 2013;30:682-90.

41. de Saint Hilaire Z, Straub J, Pelissolo A. Temperament and character in primary insomnia. Eur Psychiatry 2005;20:188-92.

42. He SS, Zhu JF, Jiang WJ, Ma J, Li GH, He YP. Sleep disturbance, negative affect and health-related quality of life in patients with maintenance hemodialysis. Psychol Health Med 2019;24:294-304.

43. Hoag JR, Tennen H, Stevens RG, Coman E, Wu H. Affect, emotion dysregulation and sleep quality among low-income women. Sleep Health 2016;2:283-8.

44. Kalmbach DA, Pillai V, Roth T, Drake CL. The interplay between daily affect and sleep: a 2-week study of young women. J Sleep Res 2014;23:636-45.

45. Kothari DJ, Davis MC, Yeung EW, Tennen HA. Positive affect and pain: mediators of the within-day relation linking sleep quality to activity interference in fibromyalgia. Pain 2015;156:540-6.

46. Lee S, Kim SJ, Park JE, Cho SJ, Cho IH, Lee YJ. Biogenetic temperament and character in insomnia and depression. J Psychosom Res 2012;72:383-7.

47. Machado L, Souza CTN, Nunes RO, de Santana CN, Araujo CF, Cantilino A. Subjective well-being, religiosity and anxiety: a crosssectional study applied to a sample of Brazilian medical students. Trends Psychiatry Psychother 2018;40:185-92.

48. Marconi S, Scarlatti F, Rizzo G, Antelmi E, Innamorati M, Pompili $M$, Brugnoli $R$, Murri MB, Amore M, Provini $F$. Is nocturnal eating in restless legs syndrome linked to a specific psychopathological profile? A pilot study. J Neural Transm (Vienna) 2015;122:1563-71.
49. Mauss IB, Troy AS, LeBourgeois MK. Poorer sleep quality is associated with lower emotion-regulation ability in a laboratory paradigm. Cogn Emot 2013;27:567-76.

50. McCrae CS, McNamara JP, Rowe MA, Dzierzewski JM, Dirk J, Marsiske M, Craggs JG. Sleep and affect in older adults: using multilevel modeling to examine daily associations. J Sleep Res 2008;17:42-53.

51. Park JH, An H, Jang ES, Chung S. The influence of personality and dysfunctional sleep-related cognitions on the severity of insomnia. Psychiatry Res 2012;197:275-9.

52. Perogamvros L, Aberg K, Gex-Fabry M, Perrig S, Cloninger CR, Schwartz S. Increased reward-related behaviors during sleep and wakefulness in sleepwalking and idiopathic nightmares. PLoS One 2015;10:e0134504. doi: 10.1371/journal.pone.0134504.

53. Rehman A, Gumley A, Biello S. Sleep quality and paranoia: the role of alexithymia, negative emotions and perceptual anomalies. Psychiatry Res 2018;259:216-22.

54. Sforza E, de Saint Hilaire Z, Pelissolo A, Rochat T, Ibanez V. Personality, anxiety and mood traits in patients with sleep-related breathing disorders: effect of reduced daytime alertness. Sleep Med 2002;3:139-45.

55. Short NA, Allan NP, Schmidt NB. Sleep disturbance as a predictor of affective functioning and symptom severity among individuals with PTSD: An ecological momentary assessment study. Behav Res Ther 2017; 97:146-53.

56. Simor P, Krietsch KN, Koteles F, McCrae CS. Day-to-day variation of subjective sleep quality and emotional states among healthy university students--a 1-week prospective study. Int J Behav Med 2015;22:625-34.

57. Simor P, Harsanyi A, Csigo K, Miklos G, Lazar AS, Demeter G. Eveningness is associated with poor sleep quality and negative affect in obsessive-compulsive disorder. J Behav Addict 2018;7:10-20.

58. Sin NL, Moskowitz JT, Whooley MA. Positive affect and health behaviors across 5 years in patients with coronary heart disease: the heart and soul study. Psychosom Med 2015;77:1058-66.

59. Sin NL, Almeida DM, Crain TL, Kossek EE, Berkman LF, Buxton OM. Bidirectional, Temporal Associations of Sleep with Positive Events, Affect, and Stressors in Daily Life Across a Week. Ann Behav Med 2017;51:402-15.

60. St George RJ, Delbaere K, Williams P, Lord SR. Sleep quality and falls in older people living in self- and assisted-care villages. Gerontology 2009;55:162-8.

61. Takano K, Sakamoto S, Tanno Y. Repetitive thought impairs sleep quality: an experience sampling study. Behav Ther 2014;45:67-82.

62. Tamagawa R, Lobb B, Booth R. Tolerance of shift work. Appl Ergon 2007; 38:635-42.

63. Theadom A, Cropley M, Humphrey KL. Exploring the role of sleep and coping in quality of life in fibromyalgia. J Psychosom Res 2007;62:145-51.

64. van de Laar M, Leufkens T, Bakker B, Pevernagie D, Overeem S. Phenotypes of sleeplessness: stressing the need for psychodiagnostics in the assessment of insomnia. Psychol Health Med 2017;22:902-10.

65. von Kanel R, Mausbach BT, Ancoli-Israel S, Mills PJ, Dimsdale JE, Patterson TL, Grant I. Positive affect and sleep in spousal Alzheimer caregivers: a longitudinal study. Behav Sleep Med 2014;12:358-72.

66. Whitehead BR, Blaxton JM. Daily well-being benefits of physical activity in older adults: does time or type matter? Gerontologist 2017;57:1062-71.

67. Altunoluk B, Bağcioğlu E, Efe E, Bahçeci B, Söylemez H. Temperament and character in differences in in patients with premature ejaculation. Noropsikiyatri Arsivi 2013;50:332-6. 
68. Fassino S, Garzaro L, Peris C, Amianto F, Pierò A, Daga GA. Temperament and character in couples with fertility disorders: a double-blind, controlled study. Fertil Steril 2002;77:1233-40.

69. Konkan R, Bayrak M, Gönüllü OG, Senormanci Ö, Sungur MZ. Sexual function and satisfaction of women with vaginismus. Dusunen Adam 2012;25:305.

70. Ozturk CS, Arkar H. Temperament and character dimensions, marital adjustment, and sexual satisfaction in couples with sexual dysfunction. Turkish J Clinical Psychiatry 2018;21:231-44.

71. Tavares IM, Laan ETM, Nobre PJ. Cognitive-affective dimensions of female orgasm: the role of automatic thoughts and affect during sexual activity. J Sex Med 2017;14:818-28.

72. Lu X, Chen Z, Cui X, Uji M, Miyazaki W, Oda M, Nagata T, Kitamura $\mathrm{T}$, Katoh T. Effects of temperament and character profiles on state and trait depression and anxiety: a prospective study of a Japanese youth population. Depress Res Treat 2012;2012:604684.

73. Haji Seyed Javadi SA. Evaluation of depression and anxiety, and their relationship with insomnia, nightmare and demographic variables in medical students. Eur Psychiatry 2017;41(Supl):S853. doi:10.1016/j. eurpsy.2017.01.1695

74. Vera-Villarroel P, Urzúa A, Jaime D, Contreras D, Zych I, Celis-Atenas K, Jaime R Silva JR, Lillo S. Positive and negative affect schedule (PANAS): psychometric properties and discriminative capacity in several Chilean samples. Eval Health Prof 2019;42:473-97.

75. Cohen JN, Taylor Dryman M, Morrison AS, Gilbert KE, Heimberg RG, Gruber J. Positive and negative affect as links between social anxiety and depression: Predicting concurrent and prospective mood symptoms in unipolar and bipolar mood disorders. Behav Ther 2017;48:820-33.

76. Littlewood D, Kyle SD, Pratt D, Peters S, Gooding P. Examining the role of psychological factors in the relationship between sleep problems and suicide. Clin Psychol Rev 2017;54:1-16.

77. Taylor ND, Fireman GD, Levin R. Trait hostility, perceived stress, and sleep quality in a sample of normal sleepers. Sleep Disord 2013;2013:735812.

78. Tsai SC, Lee-Chiong TJ. Sleep disorders and fatigue. Sleep Med Clinics 2013;8:235-9.

79. Nappier MT, Bartl-Wilson L, Shoop T, Borowski S. Sleep quality and sleepiness among veterinary medical students over an academic year. Front Veterinary Sci 2019;6:119.

80. Cloninger CR, Svrakic DM. Personality disorders. In: Sadock BJ, Sadock VA, Kaplan RP (eds). Sadock's comprehensive textbook of psychiatry. 10th ed. New York, Wolters Kluwer, 2017;5431-556.

81. Fassino S, Amianto F, Sobrero C, Abbate Daga G. Does it exist a personality core of mental illness? A systematic review on core psychobiological personality traits in mental disorders. Panminerva Med 2013;55:397-413.

82. Hajirezaei $S$, Mohammadi A, Soleimani $M$, Rahiminezhad $F$ Mohammadi MR, Cloninger CR. Comparing profile of temperament and character dimensions in patients with major depressive disorder and bipolar mood disorder and control group in the Iranian sample. Iran J Psychiatry 2017;12:147-53. 